\title{
Capturas de la raya Dasyatis longa (Myliobatiformes: Dasyatidae) en las pesquerías artesanales de Golfo Dulce, Costa Rica
}

\author{
Andrés López-Garro ${ }^{1} \&$ Ilena Zanella ${ }^{1}$ \\ 1. Asociación Conservacionista Misión Tiburón, Playas del Coco, Guanacaste, Costa Rica; alopez@misiontiburon.org; \\ izanella@misiontiburon.org
}

Recibido 11-VIII-2014. Corregido 20-XI-2014. Aceptado 22-XII-2014.

\begin{abstract}
Longtail stingray Dasyatis longa, (Myliobatiformes: Dasyatidae) catches in the artisanal fisheries of Golfo Dulce, Costa Rica. From May 2010 until May 2011, the longtail stingrays (Dasytis longa), caught by artisanal fishermen in Golfo Dulce, south Pacific of Costa Rica, were analyzed. We observed a total of 30 artisanal fishing operations in different areas of Golfo Dulce, with muddy bottoms and very shallow waters (less than 30m). The areas called "Los Bajos" and "Pique Fijo" represented the $26.7 \%$ and $23.3 \%$ of all the operations, respectively. Other important areas were "La Cienaga" (20.0\%), "Lapa-Ríos" (6.7\%) "Piedra del Sombrero" (6.7\%) and "Pavones" (6.7\%). "Matapalo", "Corcovado" and "Punta Banco" were less used by fishermen (9.9\% of trips). In the captures, 345 (39.6\%) were sharks (Sphyrnidae Carcharhinidae, Heterodontidae, Ginglymostomatidae and Triakidae), 228 (26.1\%) bait fish (Aridae), 112 (13.1\%) rays (Dasyatidae, Myliobatidae, Rhinobatidae and Mobulidae) and 111 (12.7\%) commercial fish (Lutjanidae, Serranidae, Sciaenidae). The most abundant ray was $D$. longa, with $75.7 \%$ of total rays caught, $\mathrm{n}=112$ ), and more than $10 \%$ of total catch. The longtail stingrays male: female ratio was $1.33: 1$. Mean width disc length was $84.63 \pm 12.11 \mathrm{~cm}$. The highest Catch per unit effort, CPUE, was in January-February (0.006 stingrays per hook per hour). The lowest was in July and August (0.0004). Nearly all rays caught were released. We recommend regulations on the artisanal bottom line during from January to February in the areas of Los Bajos, Pique Fijo, and La Cienega. Rev. Biol. Trop. 63 (Suppl. 1): 319-327. Epub 2015 April 01.
\end{abstract}

Key words: Golfo Dulce, Costa Rica, longtail stingray, Dasyatis longa, CPUE, artisanal fishermen.

En las pesquerías artesanales del Pacífico de Costa Rica se capturan de manera incidental alrededor de 30 diferentes elasmobranquios (15 especies de tiburones y 15 de rayas) (Arauz, López \& Zanella, 2007; López, Arauz, Zanella $\&$ Le Foulge, 2009). Sobresalen tiburones de las familias Carcharhinidae, Triakidae y Sphyrnidae, que son capturados en zonas donde los pescadores buscan especies comerciales como los pargos (Lutjanidae), cabrillas (Serranidae) y congrios (Ophidiidae). A su vez, las rayas más comunes en las capturadas artesanales son rayas de las familias Dasyatidae, Myliobatidae, Rhinobatidae y Mobulidae (Arauz et al., 2007; López et al., 2009; Amador, 2010).

El bajo valor económico y la limitada comercialización que han tenido las rayas en muchas de las comunidades costeras costarricenses, ocasiona que la mayoría de las veces que son capturadas sean liberadas por los pescadores. Sin embargo esta situación ha comenzado a cambiar, principalmente en comunidades cercanas a centros pesqueros como Puntarenas, Golfito, Cuajiniquil, donde algunas especies de rayas se comercializan a bajos precios para mercados internacionales. Tal es el caso de la raya látigo (Dasyatis longa Garman, 1880), cuya carne ha comenzado a comercializarse para su exportación a México (Amador, 2010). Su gran tamaño y peso, hacen de esta especie una de las más atractiva para el comercio. Esta misma situación se reporta para el Pacífico ecuatoriano, donde $D$. longa en los últimos años se ha convertido en una de las 
cinco especies de elasmobranquios más capturada por la demanda de su carne y sus espinas utilizadas en la elaboración de artesanías (Guerrón, 2007).

Es preocupante el incremento en la comercialización de la carne de raya látigo en la región, ya que por su biología (alta longevidad, crecimiento lento, madurez tardía y baja fecundidad) sus poblaciones no tienen la capacidad de soportar la presión pesquera permanente y mucho menos la capacidad de recuperarse en caso de que la población sea sobreexplotada (Stevens, Bonfil, Dulvey \& Walker, 2000). Por ejemplo, en la comunidad de Tárcoles, en el Pacífico Central de Costa Rica, Amador (2010) reporta que luego de tres años de extracción intensa, disminuyeron en un $60 \%$ las capturas de $D$. longa descargadas por pescadores artesanales, debido sobre todo a una pesca dirigida.

Dasyatis longa es una especie costera que habita fondos arenosos, lodosos y rocosos de esteros, bahías y golfos desde California hasta Ecuador (Arauz et al., 2007; Robertson \& Allen, 2008; López et al., 2009; Zanella, Lopez \& Arauz, 2009). Sus hábitos bentónicos, su gran tamaño y su preferencia por hábitats de aguas costeras, hacen que $D$. longa, además de ser común en la pesquerías, sea una de las rayas más avistadas durante buceos Scuba en la costa del Pacífico de Costa Rica, incluido el Golfo de Papagayo (Arauz, López, Zanella \& Bolaños, 2009). Por lo anterior, a pesar de ser una especie de bajo valor comercial pesquero, D. longa tiene un alto valor económico a nivel de turismo y buceo, ya que los avistamientos de tiburones y rayas son cada vez más atractivos para los buzos (Arauz et al., 2009). En la actualidad $D$. longa se encuentra incluida en la Lista Roja de la UICN como una especie cuyas poblaciones cuentan con datos deficientes para evaluar su estado de conservación.

A fin de promover una pesquería más responsable de la raya látigo en Golfo Dulce, este trabajo caracterizó las capturas incidentales de $D$. longa de pescadores artesanales que realizan faenas con línea de fondo. Con los resultados obtenidos se recomiendan estrategias de manejo para algunas zonas de Golfo
Dulce en los meses donde la abundancia de D. longa fue mayor.

\section{MATERIALES Y MÉTODOS}

Área de estudio: El Golfo Dulce se ubica en el Pacífico Sur de Costa Rica, entre las coordenadas $08^{\circ} 00^{\prime}$ y $09^{\circ} 00^{\prime} \mathrm{N}-82^{\circ} 30^{\prime}$ y $84^{\circ} 00^{\prime}$ $\mathrm{W}$ de la provincia de Puntarenas. Es un Área Marina de Pesca Responsable (AMPR), que se divide según su decreto de creación (Acuerdo A.J.D.I.P. 191-2010) por sus características geomorfológicas, ecológicas y la información científica y la brindada por los pescadores, en tres áreas de pesca: Golfo Dulce Externo (Zona A), Interno (Zona B) y la zona de Golfito. Con base en esta división pesquera y las regulaciones actuales del AMPR Golfo Dulce, los datos analizados sobre las capturas de la raya látigo (D. longa) provienen de faenas pesqueras realizadas en la Zona Externa o Zona A de Golfo Dulce y sus alrededores. Sobresalen zonas costeras cercanas a diferentes comunidades, playas y ríos conocidas por los pescadores como Jiménez-Puntarenitas, Zancudo, Pilón-Manzanillo, Punta Banco y Matapalo, entre otros.

Descripción pesquerías artesanales en Golfo Dulce: Los pescadores artesanales de algunas comunidades de Golfo Dulce tienen como objetivo principal especies comerciales como los pargos (Lutjanidae), cabrillas (Serranidae) y congrios (Ophidiidae), entre otras especies. Para este fin, se utiliza principalmente la línea de fondo, la cual consta de una línea madre de nylon de aproximadamente $1-3 \mathrm{~km}$ de largo, de la que cuelgan anzuelos tipo circulares $\left(\mathrm{N}^{\mathrm{o}} 10\right.$ y 12$)$ y $\mathrm{J}\left(\mathrm{N}^{\mathrm{o}} 7\right.$ y 8$)$ cada 5-10m. Aunque los pescadores dirigen sus esfuerzos hacia especies comerciales, también se capturan de manera incidental importantes cantidades de tiburones y rayas (López, 2012).

Información biológica: La información biológica referente a las capturas de la raya látigo en faenas pesqueras artesanales realizadas en Golfo Dulce, fue recolectada mediante la observación a bordo de embarcaciones en un ciclo anual (mayo 2010 a 2011). En cada una 
de las observaciones a bordo, se registraron las coordenadas geográficas o zona de pesca por medio de un Sistema de Geoposicionamiento Global (GPS), las horas exactas de pesca, el número y tipo de anzuelos calados y un registro del total de individuos por especie de la captura comercial y su condición de captura (vivo o muerto). Luego de su identificación taxonómica, se registró el sexo (según la presencia o ausencia de hemipenes) y se recolectó la anchura de disco (ADi) de los individuos de $D$. longa. Este parámetro se define como la distancia comprendida entre los extremos de las aletas pectorales $( \pm 0.1 \mathrm{~cm})$.

Abundancia relativa: $\mathrm{La}$ abundancia relativa de $D$. longa fue estimada a partir de la información científica recolectada durante las faenas de pesca (línea de fondo) y observador a bordo. La Captura por Unidad de Esfuerzo $\left(\mathrm{CPUE}_{1000}\right)$ se expresó como el número de individuos de $D$. longa capturados por cada 1000 anzuelos (Tavares, 2005). No obstante, para estudiar la fluctuación de la abundancia relativa a través del tiempo, se consideró incluir en la función el tiempo efectivo que los anzuelos estuvieron en el agua, el cual puede variar entre temporadas, entre pescadores e inclusive entre faenas de pesca (Musick \& Bonfil, 2004; López et al., 2009).

Se utilizó la siguiente ecuación para calcular el número de tiburones o rayas capturados por anzuelo, por hora:

$$
\begin{gathered}
\mathrm{CPUE}=\left(\left(\mathrm{T}_{\text {D.longa }}\right) / \mathrm{TA}\right) \\
{[(\mathrm{HIV}-\mathrm{HIC}) / 2+(\mathrm{HFV}-\mathrm{HFC}) / 2]}
\end{gathered}
$$

donde

$\mathrm{T}_{\text {D. longa }}=$ Total de individuos de $D$. long $a$

$\mathrm{TA}=$ total de anzuelos

$\mathrm{HIV}=$ hora inicial virado

$\mathrm{HIC}=$ hora inicial calado

$\mathrm{HFV}=$ hora final virado

$\mathrm{HFC}=$ hora final calado

Los valores de CPUE medios calculados para Golfo Dulce, fueron graficados para la raya látigo a lo largo del año.
Análisis de datos biológico-pesqueros de observaciones abordo: Los valores de ancho de disco de los individuos de $D$. longa provenientes de las observaciones abordo fueron promediados (media); además, se calculó el mínimo (min), máximo (max) y la desviación estándar (DE). Con los valores de ancho de disco (ADi) se calculó la distribución de frecuencia para las clases de rayas látigo más importantes.

Proporción de sexos: La proporción de sexo de las rayas látigo analizadas se estimó según la relación de machos y hembras identificados en las capturas. Se utilizó un análisis de varianza no paramétrico de una vía (KruskallWallis) para determinar diferencias entre la longitud total de machos y hembras utilizando el programa InfoStat (Di Rienzo et al., 2008).

Cuando la condición de captura y del ambiente lo permitió, a las rayas látigo capturadas se les implantó una marca convencional tipo "flexi" en la aleta pectoral, lo anterior, para conocer sobre el uso de hábitats y los movimientos de $D$. longa en Golfo Dulce y alrededores.

\section{RESULTADOS}

De mayo 2010 a mayo 2011 se recolectó información biológica-pesquera referente a 30 faenas pesqueras artesanales con línea de fondo en la zona externa de Golfo Dulce y sus alrededores. Destacan las zonas conocidas por los pescadores como Los Bajos y Pique Fijo con el $26.7 \%$ y $23.3 \%$ respectivamente, de todas las faenas pesqueras realizadas. Otras zonas importantes fueron La Ciénaga (20.0\%), LapaRíos (6.7\%), Piedra del Sombrero (6.7\%) y Pavones (6.7\%). Zonas del Golfo Dulce como, Matapalo, Punta Banco y Corcovado fueron menos frecuentadas por los pescadores, ya que representaron en conjunto el $9.9 \%$ de las faenas analizadas.

En las 30 faenas con observador abordo analizadas, los pescadores utilizaron líneas de fondo con una longitud y número promedio de anzuelos de $2.1 \pm 0.8 \mathrm{~km}$ y $308.1 \pm 90.5$ anzuelos, respectivamente. 
Durante las 30 faenas de pesca artesanal se capturaron 872 organismos: de estos, $345(39.6 \%)$ fueron tiburones (Sphyrnidae, Carcharhinidae, Heterodontidae, Ginglymostomatidae y Triakidae), 228 (26.1\%) peces clasificados como carnada (Aridae), 112 (13.1\%) rayas (Dasyatidae, Myliobatidae, Rhinobatidae y Mobulidae) y 111 (12.7\%) peces comerciales (Lutjanidae: Serranidae y Scianidae).

Del total de las 112 rayas analizadas en las observaciones abordo, la especie más abundante fue $D$. longa, con el $75.7 \%(\mathrm{n}=84)$ del total de rayas capturadas, seguida por la raya gavilana Aetobatus narinari con $12.6 \%$ y la raya nariz de vaca (Rhinoptera steindachneri) con $5.4 \%$. Otras rayas como la guitarra (Rhinobatos. leucorhynchus), la raya de puntos (Urotrygon chilenis), la mobula (Mobula sp.) y la raya mariposa (Gymnura marmorata), estas dos últimas fueron esporádicas y aportaron entre ambas menos del 4\% del total (Fig. 1).

Los análisis de captura a bordo de embarcaciones de pesca artesanal en Golfo Dulce, revelan rayas látigo en la mayoría de las faenas, aportando en total más del $10 \%$ de todos organismos capturados. Además más del $95 \%$ de las rayas látigo analizadas se capturaron en zonas de pesca de Golfo Dulce con fondos lodosos y de baja profundidad (menos de $30 \mathrm{~m})$. Sobresalen las zonas conocidas como Los Bajos, Pique Fijo, Punta Banco y La Ciénaga-Jiménez (Cuadro 1).

\section{CUADRO 1}

Abundancia de rayas látigo (Dasyatis longa) por zona de pesca durante el ciclo anual (Mayo 2010-Mayo 2011) en Golfo Dulce. Costa Rica

\section{TABLE 1}

Abundance of longtail stingray (Dasyatis longa) observed by fishing zones, during the annual period (May 2010-May 2011) in Golfo Dulce, Costa Rica

\begin{tabular}{lc}
\multicolumn{1}{c}{ Zona de pesca } & Número de $D$. longa \\
Los Bajos & 18 \\
Pique Fijo & 51 \\
Punta Banco & 4 \\
Jiménez-La Ciénaga & 9 \\
Pavones & 0 \\
Corcovado & 2 \\
Piedra del sombrero & 0 \\
Matapalo & 0 \\
Total & $\mathbf{8 4}$ \\
\hline
\end{tabular}

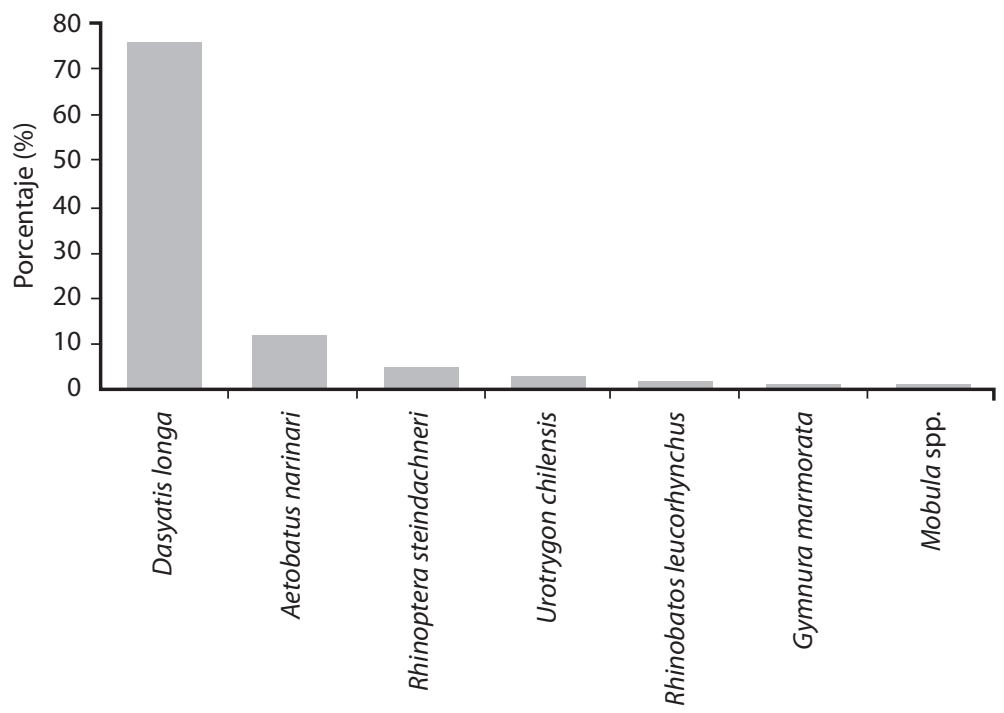

Fig. 1. Total de rayas por especie capturadas durante los 30 viajes de observación abordo ( $\mathrm{n}=112)$. Golfo Dulce. Costa Rica, 2010-11.

Fig. 1. Total of rays by species caught during the 30 onboard observations (n=112). Golfo Dulce. Costa Rica, 2010-11. 
Con respecto a la proporción sexual, los análisis revelaron una proporción sexual de de 1.33 machos por cada hembra, siendo esta diferencia significativa estadísticamente $(\mathrm{H}=40.38$; $\mathrm{p}<0.001)$. A su vez, la longitud media del ancho del disco para las rayas látigo analizadas fue de $84.63 \pm 12.11 \mathrm{~cm}$, sin embargo durante las observaciones abordo se analizaron $D$. longa con longitudes de disco desde los $60 \mathrm{~cm}$ hasta los $119 \mathrm{~cm}$.

La distribución de frecuencia de ancho de disco para las rayas látigo analizadas, muestra que la mayoría de las $D$. longa capturadas (aproximadamente el 60\%) obtuvo longitudes comprendidas entre los 60 y $80 \mathrm{~cm}$. A su vez, menos del $30 \%$ de las rayas látigo tenían longitudes comprendidas entre los 90 y $119 \mathrm{~cm}$ (Fig. 2).

Las CPUE ${ }_{1000}$ de D. longa en las faenas con línea de fondo a lo largo del año reveló un promedio de $11.8 \pm 22.42$ rayas látigo por cada 1000 anzuelos. Los análisis de abundancia relativa expresada como la captura por unidad

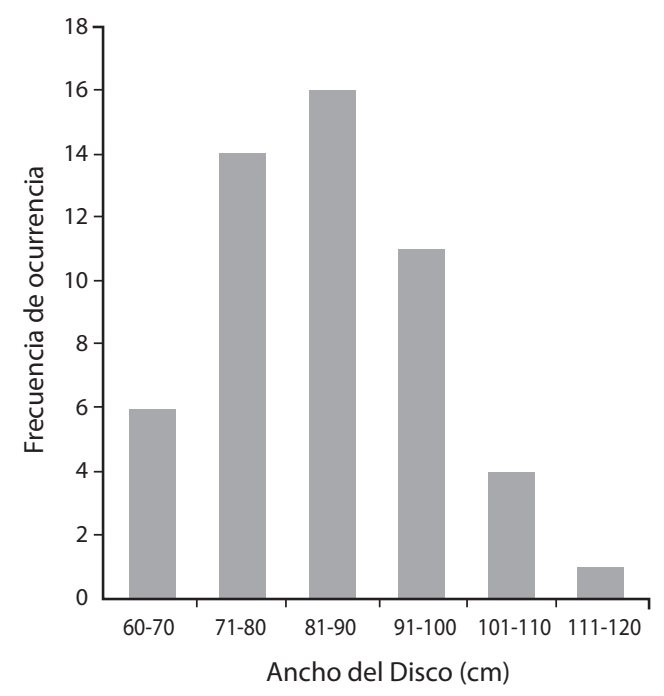

Fig. 2. Distribución de tallas de las raya látigo (Dasyatis longa), capturadas con línea de fondo durante faenas de pesca realizadas en Golfo Dulce, Costa Rica 2010-11 $(\mathrm{n}=52)$.

Fig. 2. Width disc length frequency distribution for the longtail stingray (Dasyatis longa) caught with bottom line during the onboards fisheries observations in Golfo Dulce, Costa Rica, 2010-11. de esfuerzo (CPUE) por anzuelo por hora muestran cambios en la abundancia de $D$. longa en diferentes meses del año con dos importantes picos. El más fuerte en el bimestre de enero y febrero, meses donde se obtuvieron las mayores CPUE con un promedio de 0.006 rayas látigo por anzuelo por hora. El segundo pico se presentó en los meses de Octubre y Noviembre donde se capturaron aproximadamente 0.0035 $D$. longa por anzuelo por hora. De manera inversa en los meses de julio y agosto las rayas látigo presentaron la menor CPUE en las faenas pesqueras, con un promedio de 0.0004 rayas por anzuelo por hora (Fig. 3).

Finalmente, la condición de captura, el bajo valor comercial y la disponibilidad de los pescadores permitió liberar con vida y libres de anzuelo el 99\% de las rayas de las diferentes especies capturadas. Respecto a $D$. longa, se destaca que se marcaron convencionalmente 54 individuos. (Fig. 4).

\section{Recapturas pesqueras de rayas láti- go marcadas convencionalmente en Golfo Dulce: Del total de individuos marcados, dos fueron recapturados. El primero de ellos fue marcado en la localidad de los Bajos (cercanías de Puerto Jiménez) y fue capturado después de ocho meses a más de 150 millas de distancia en las cercanías a Cabo Blanco en el Pacífico Cen- tral de Costa Rica. El segundo individuo fue recapturado un año después a una o dos millas de distancia de donde fue marcado y liberado (Puerto Jiménez).}

\section{DISCUSIÓN}

Los hábitos bentónicos de la mayoría de las especies de rayas hacen que sean capturadas comúnmente en las pesquerías artesanales realizadas en aguas costeras como sucede en Golfo Dulce, donde más del 13\% de la captura total fueron rayas. En Golfo Dulce D. longa representó más del $75 \%$ de todas las rayas capturadas abordo y del $10 \%$ de la captura total. De la misma forma, en las pesquerías del Golfo de California especies del género Dasyatis (D. longa y D. brevis) figuran con 


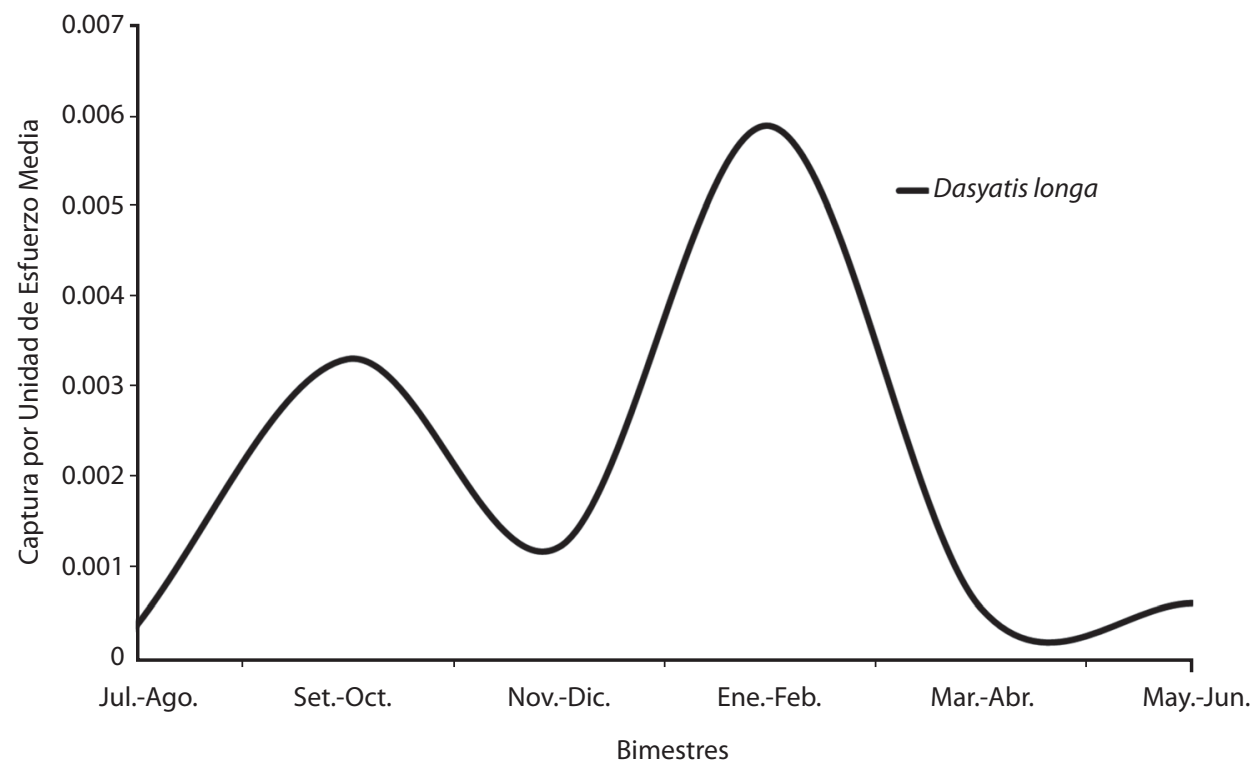

Fig. 3. Abundancia relativa anual expresada como CPUE para las rayas látigo en bimestres. Golfo Dulce, Costa Rica. 2010-11.

Fig. 3. Annual relative abundance for the longtail stingray (Dasyatis longa), as Catch per Unit Effort by bimester. Golfo Dulce, Costa Rica. 2010-11.

importantes capturas en la zona costera de Sinaloa (Villavicencio, 1995). En el Golfo de México, Ramírez-Mosqueda, Pérez-Jiménez y Mendoza-Carranza (2012) reportan a la raya látigo americana $D$. americana como el elasmobranquio más capturado y desembarcado por flotas artesanales en el sur del Golfo de México. A su vez, la raya látigo-hocicona (D. guttata) constituye la principal captura de la flota pesquera en Boca de Río, Venezuela, representando el $48,94 \%$ del total de las especies de rayas capturadas (Cordovés, Ron \& Tavares, 2009). En aguas costarricense, en la parte externa del Golfo de Nicoya, Amador (2010) reportó a D. longa como una raya abundante y de importancia en las pesquerías artesanales de Tárcoles por su reciente valor comercial.

En Golfo Dulce la raya látigo fue capturada sobre todo (más del 95\%) en faenas pesqueras realizadas en fondos someros y lodosos como La Ciénaga, Los Bajos y Pique Fijo. Esto se debe a que $D$. longa prefiere estos hábitats costeros que le brindan alimento, lo cual también ha sido reportado en el Golfo de Nicoya por Zanella et al., (2009) y Amador (2010).

López (2009) reporta que $D$. longa en el Pacífico colombiano alcanza la madurez

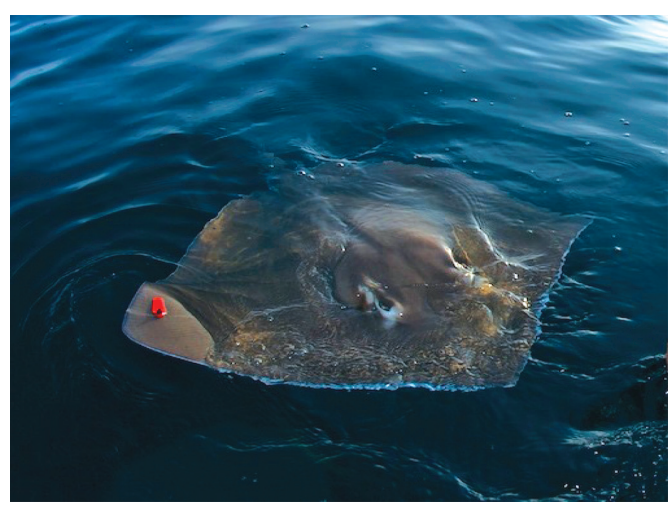

Fig. 4. Raya látigo (Dasyatis longa) liberada y marcada con una marca convencional "flexi" en Golfo Dulce, Costa Rica 2010-11. Fotografía: David García/Misión Tiburón, 2012.

Fig. 4. Longtail stingray (Dasyatis longa) tagged and released with a conventional flexi-tag in Golfo Dulce, Costa Rica. 2010-11. Picture: David García/Misión Tiburón, 2012. 
sexual a los 92 y $120 \mathrm{~cm}$ de ancho de disco para machos y hembras, respectivamente. Así mismo, Villavicencio, Downton, y Meléndez (1994) en Baja California Sur determinaron la talla mínima de madurez de los machos y hembras de $D$. longa en 80 y $110 \mathrm{~cm}$ de ancho de disco respectivamente. De acuerdo a lo anterior, las capturas de esta especie en Golfo Dulce están constituidas por un elevado porcentaje (más del 90\%) de individuos inmaduros o juveniles, que aún no han alcanzado la primera madurez sexual, ya que la mayoría de individuos presentó un rango entre los 60 y $119 \mathrm{~cm}$ de ancho de disco, con un promedio de $85 \mathrm{~cm}$ (Fig. 2). Con respecto a la proporción de sexos en las capturas, en Golfo Dulce dominaron los machos de $D$. longa $(\mathrm{H}=40.38$ y $\mathrm{p}<0.001)$. A diferencia de lo encontrado en Tárcoles, Costa Rica por Amador (2010), quien reporta proporciones sexuales para $D$. longa dominadas por hembras maduras y lo reportado por López (2009) en el Pacífico colombiano, quien registró, proporciones similares hembra: macho. Por lo anterior, el comportamiento en Golfo Dulce podría deberse a la agregación de individuos machos de $D$. longa con fines de alimentación, sin embargo solo estudios a largo plazo podrían confirmar esta hipótesis.

El limitado comercio de esta especie en Golfo Dulce y la alta sobrevivencia de las rayas en las líneas de fondo hizo posible liberar el $99 \%$ de las rayas capturadas. Al no haber demanda, los pescadores prefieren liberarlas, sin embargo en otras comunidades de Costa Rica en el Golfo de Nicoya, las rayas son comercializadas para exportar su carne a países de la región como México. Amador (2010) reporta en la comunidad de Tárcoles la utilización por pescadores artesanales de una línea de fondo especializada provista de anzuelos de gran tamaño para la captura de $D$. longa Inclusive, esta investigadora describió como la pesca dirigida hacia esta especie, en solo tres años produjo un descenso de las capturas cercano al $60 \%$, lo cual pudo afectar a la población de manera irreversible, apoyado esto en que menos del $30 \%$ de las hembras capturadas durante su investigación había alcanzado la madurez sexual.

La alta sobrevivencia de los individuos de la raya látigo capturadas por los pescadores, está relacionada de forma directa con los hábitos ecológicos de la especie, ya que $D$. longa, al igual que muchos otros tiburones y rayas demersales, posee espiráculos que le ayudan a maximizar el oxígeno durante la respiración, por esta razón no tienen que nadar constantemente (Carlson, Goldman \& Lowe, 2004). Además, los individuos de $D$. longa oxigenan sus branquias con un sistema de bombeo en el área bucal. De esta forma, cuando son enganchados por un anzuelo, logran mantenerse con vida por más tiempo. Sin embargo, su sobrevivencia a largo plazo depende en mayor grado del método de liberación y por ende del pescador.

Los análisis de abundancia relativa (CPUE) muestran las mayores capturas pesqueras de $D$. longa entre enero y febrero y las menores entre julio y agosto. Lo anterior concuerda con lo reportado por Amador (2010) en la comunidad de Tárcoles en el año 2007, cuando los pescadores artesanales descargaron la mayor cantidad de rayas látigo en enero y febrero $(2.2 \mathrm{~kg} / \mathrm{h}$ y $4.2 \mathrm{~kg} / \mathrm{h}$ respectivamente). De la misma manera los reportes de avistamientos subacuáticos en sitios de buceo del Golfo de Papagayo en Costa Rica, reportan las mayores abundancias de $D$. longa durante enero, febrero y marzo. En estos meses las aguas del Pacífico Norte costarricense están expuestas a fuertes vientos y oleajes que vuelven las aguas más frías y productivas atrayendo diferentes especies marinas, entre estas la raya látigo (Arauz et al., 2009). Este fenómeno es conocido como afloramientos o surgencias (intercambio entre las aguas profundas frías y ricas en nutrientes y las aguas superficiales menos productivas). A la vez, los afloramientos causan un drástico enfriamiento de las aguas en el Pacífico Norte en los meses de diciembre, enero y febrero, promoviendo el desarrollo de toda la red trófica, que incluye desde organismos plantónicos, como larvas de peces e invertebrados, hasta 
grandes pelágicos como ballenas y tiburones (Brenes, Kwiecinski, Croz \& Chaves, 1995).

Además López (2012) reportan como en Golfo Dulce, las rayas látigo y los tiburones martillo (Sphyrna lewini) presentan CPUE opuestas. De tal forma, cuando se obtuvieron las mayores CPUE de D. longa, casi no se capturaron tiburones martillo. Lo anterior ha sido reportado para otras zonas como en el Pacífico Central de México, donde Pérez-Jiménez, Sosa-Nishizak, y Estrada (2005) reportan las mayores capturas de D. longa, D. brevis y $R$. glaucostigma, cuando los tiburones son poco abundantes. Esta tendencia podría deberse a cambios estacionales en las variables ambientales, por ejemplo Arauz et al. (2009) reportan las mayores abundancia de D. longa en los meses de enero y febrero, meses donde la temperatura del agua baja hasta los $14^{\circ} \mathrm{C}$ en el Golfo de Papagayo, Pacífico Norte de Costa Rica.

Los cambios en las abundancias relativas de $D$. longa a lo largo del año y las recapturas de individuos marcados, sugiere que esta especie realiza migraciones tanto dentro como fuera de Golfo Dulce. Por lo anterior y debido a que en otras zonas del Pacífico D. longa es capturada comercialmente, es importante promover estrategias de manejo para su conservación a lo largo de toda la costa pacífica de Costa Rica. Debido a la alta sobrevivencia y capturas de $D$. longa durante enero-febrero en las pesquerías artesanales, se recomienda regular la línea de fondo en la zona externa de Golfo Dulce en estos meses, sobre todo en sitios con fondos lodos de poca profundidad (menos de $30 \mathrm{~m}$ ) como La Ciénaga y Los Bajos. Además debido a que la mayoría de los organismos capturados eran juveniles y a que en la actualidad $D$. longa no se comercializa en Golfo Dulce, se propone prohibir la captura, transporte y comercialización de esta especie. Por último, la importancia ecológica y turística (buceo recreativo) de $D$. longa en la actualidad, justifican la necesidad de desarrollar más investigación, a fin de identificar hábitats críticos o esenciales y conocer mejor sobre el uso de hábitat y movimientos de esta especie en la zona costera.

\section{AGRADECIMIENTOS}

Este trabajo de investigación con la raya látigo fue posible solo por el apoyo de diferentes pescadores artesanales de Golfo Dulce, en especial de Puerto Jiménez y Pavones. Gracias a José Collado, Pedro Rodríguez, Pedrito Rodríguez, William Mata, por su cooperación y por permitirnos analizar abordo las capturas de rayas de sus faenas artesanales. Además debemos agradecer por su apoyo financiero a Conservación Internacional Centroamérica, Columbus Zoo and Aquarium, Mohamed Bin Zayed Conservation Fund, Marine Action Conservation Fund (MACF). Por último agradecer. A Maurizicio Protti de la Universidad Nacional de Costa Rica, por sus sugerencias que mejoraron el artículo. Se agradece el Proyecto Consolidación de Áreas Marinas Protegidas del Sistema Nacional de Áreas de Conservación (SINAC), Programa de Naciones Unidas para el Desarrollo (PNUD) y el Global Environment Facility (GEF) por su colaboración en la publicación.

\section{RESUMEN}

Entre mayo 2010 y mayo 2011 se analizaron las rayas látigo (Dasytis longa) capturadas por pescadores artesanales de Golfo Dulce, Pacífico Sur de Costa Rica. En total se realizaron 30 faenas en diferentes zonas con fondos lodosos y rocosos de baja profundidad (menos de $30 \mathrm{~m}$ ). Durante las 30 faenas de pesca se capturaron 872 organismos, de estos, $112(13.1 \%)$ fueron rayas. La especie más abundante fue $D$. longa, aportando el $75.7 \%$ de las rayas y más del $10 \%$ de la captura total. Los análisis revelaron una proporción sexual de 1.33 machos por cada hembra para las rayas látigo y una longitud media del ancho del disco de $84.63 \pm 12.11 \mathrm{~cm}$. Con respecto a la captura por unidad de esfuerzo (CPUE), las mayores CPUE se obtuvieron en enero y febrero que se capturaron en promedio de 0.006 rayas látigo por anzuelo por hora. La condición de captura, el bajo valor comercial y la disponibilidad de los pescadores, permitió liberar el 99\% de las rayas capturadas. Debido a la alta sobrevivencia y capturas de D. longa en las pesquerías artesanales, se recomienda regular la línea de fondo durante enero-febrero en Los Bajos, Pique Fijo y La Ciénaga (Zona externa Golfo Dulce).

Palabras clave: Golfo Dulce, Costa Rica, raya látigo, Dasyatis longa, CPUE, pesquerías artesanales. 


\section{REFERENCIAS}

Amador, L. (2010). Descripción y análisis de la pesca artesanal de la raya látigo Dasyatis longa (Dasyatidae) en Tárcoles, Puntarenas, Pacífico de Costa Rica (Tesis de licenciatura). Escuela de Biología, Universidad Nacional de Costa Rica, Heredia, Costa Rica.

Arauz, R., López, A., \& Zanella, I. (2007). Análisis de la descarga anual de tiburones y rayas en la pesquería pelágica y costera del Pacífico de Costa Rica (Playas del Coco, Tárcoles y Golfito) (Informe final). San José, Costa Rica: Pretoma para Conservación Internacional.

Arauz, R., López, A., Zanella, I., \& Bolaños, A. (2009). Acción por los tiburones: Análisis de las capturas y avistamientos de elasmobranquios en las aguas costeras y pelágicas del Pacífico de Costa Rica (Informe final). San José, Costa Rica: Pretoma para Conservación Internacional.

Brenes, C., Kwiecinski, B., Croz, L., \& Chaves, J. (1995). Características oceanográficas de la plataforma continental de América Central y aguas adyacentes (Informe de campañas). Panamá, PRADEPESCA.

Carlson, J. K., Goldman, K. J., \& Lowe, C. G. (2004). Metabolism, Energetic demand and Endothermy. In Carrier, J. C., J. A. Musick \& M. R. Heithaus (Eds.), Biology of sharks and their relatives (pp. 203-224). Boca Raton, Florida, USA: CRC Press.

Cordovés, M., Ron, E., \& Tavares, R. (2009). Composición de las capturas comerciales de la raya látigo-hocicona, Dasyatis guttata (Bloch \& Schneider, 1801), procedentes de la pesca artesanal en la Isla de Cubagua, Venezuela. Memoria del Gulf and Caribbean Fisheries Institute, 62, 567-570.

Di Rienzo, J. A, Casanoves, F., Balzarini, M., Gonzalez, L., Cuadroda, M., \& Robledo, C. (2008). InfoStat (Versión 2008). Argentina: Grupo InfoStat, FCA, Universidad Nacional de Córdoba.

Guerrón, J. (2007). La nueva pesquería de rayas en Puerto López, zona de Amortiguamiento del Parque Nacional Machalilla (Proyecto de Baccalaureus Scientiae) Universidad San Francisco, Quito, Ecuador.

López, J. (2009). Aspectos Biológicos de la raya látigo Dasyatis longa (Pisces: Dasyatidae) de la zona central del Pacífico colombiano (Trabajo para optar al título de biólogo). Universidad del Valle, Santiago de Cali, Colombia.

López, A., R., Arauz, R., Zanella, I., \& Le Foulge, L. (2009). Análisis anual de las capturas de tiburones y rayas en las pesquerías artesanales de Tárcoles, Pacífico Central de Costa Rica. Revista Ciencias Marinas y Costeras, 1, 145-157.

López, A. (2012). Identificación, evaluación y manejo de hábitats críticos utilizados por el tiburón martillo, Sphyrna lewini, y otros elasmobranquios en Golfo Dulce, Costa Rica (Tesis de maestría). Universidad Nacional, Heredia, Costa Rica.

Musick, J., \& Bonfil, R. (2004). Elasmobranch fisheries management techniques (Report APEC Fisheries Working Group). Singapore, Japan.

Pérez-Jiménez, J. C., Sosa-Nishizak, O., \& Estrada E. F. (2005). Artisanal Shark Fishery at "Tres Marias" Islands and Isabel Island in the Central Mexican Pacific. Journal of Northwest Atlantic Fishery Science, 35, 333-343.

Ramírez-Mosqueda, E., Pérez-Jiménez, J. C., \& MendozaCarranza, M. (2012). Reproductive parameters of the southern stingray Dasyatis americana in southern gulf of Mexico. Latin America Journal of Aquatic Research, 40(2), 335-344.

Robertson, D. R., \& Allen, G. R. (2008). Shorefishes of the tropical Eastern Pacific online information system. Retrived from http:/www.stri.org/sftep

Stevens, J. D., Bonfil, R., Dulvey, N. K., \& Walker, P. A. (2000). The effects of fish on sharks, rays, and chimeras (Chondrichthyans), and the implications for marine ecosystems. Journal of Marine Science, $57,476-494$.

Tavarez, R. (2005). Abundance and distribution of sharks in Los Roques Archipelago National Park and other Venezuelan oceanic islands 1997-1998. Ciencia $y$ Mar, 31, 441-454.

Villavicencio, C., Downton, C., \& Meléndez, E. (1994). Tamaño y reproducción de la raya Dasyatis longus (Pisces: Dasyatidae), en Bahía Almejas, Baja California Sur, México. Revista de Biología Tropical, 42, 375-377.

Villavicencio, C. J. (1995). Distribución temporal y condición reproductiva de las rayas (Pisces: Batoidei), capturadas comercialmente en Bahía Almejas, B.C.S., México. Revista de Investigación Científica, 6, 1-12.

Zanella, I., Lopez, A., \& Arauz, R. (2009). Caracterización de las descargas del tiburón martillo, Sphyrna lewini, en un área de crianza ubicada en el Golfo de Nicoya, Costa Rica. Revista de Ciencias Marinas y Costeras, 1,175-195. 
